\title{
Double optimal stopping of a risk process
}

\author{
ANNA KARPOWICZ† and KRZYSZTOF SZAJOWSKI* \\ Institute of Mathematics and Computer Science, Wroclaw University of Technology, Wybrzeże, \\ Wyspiańskiego 27, Wroclaw, Poland
}

(Received 28 April 2006; in final form 15 October 2006)

\begin{abstract}
The following problem in risk theory is considered. An insurance company, endowed with an initial capital $a>0$, receives insurance premiums and pays out successive claims. The losses occur according to renewal process. At any moment, the company may broaden or narrow down the offer, what entails the change of the parameters. This change concerns the rate of income, the intensity of renewal process and the distribution of claims. After the change, the management wants to know the moment of the maximal value of the capital assets. Therefore, our goal is finding two optimal stopping times: the best moment of change the parameters and the moment of maximal value of the capital assets. We will use a dynamic programming method to calculate the expected capital at that times.
\end{abstract}

Keywords: Risk reserve process; Optimal stopping; Dynamic programming; Capital assets; SemiMarkov process; Infinitesimal generator

Mathematics Subject Classification 2000: Primary 60G40; 60K99; Secondary 90A46

\section{Introduction}

In this paper, the following problem is considered. The insurance company, endowed with an initial capital $a>0$, receives insurance premium with constant rate of income $c_{1}$ and pays out successive claims, which are representing by i.i.d. random variables $X_{1}, X_{2}, \ldots$ with cumulative distribution function $H_{1}$. The losses occur according to the renewal process $\left\{N_{1}(t), t \geq 0\right\}$, so that $N_{1}(t)$ is the number of claims up to the time $t$. The renewal process is independent on the sequence of claims. Let $T_{i}$ denote the moment of the $i$ th claim $\left(T_{0}=0\right)$, then the random variables $S_{i}=T_{i}-T_{i-1}$ are i.i.d. with continuous cumulative distribution function $F_{1}$. Let us assume that the company can change the parameters of the classical risk model at any time $s$. It corresponds with the circumstances when companies broaden the offer to appeal to a wider range of customers. Then the rate of income changes to $c_{2}$ and the losses, after the time $s$, occur according to another renewal process $\left\{N_{2}(t), t \geq 0\right\}$. The claims after the change are i.i.d. random variables $\tilde{X}_{1}, \tilde{X}_{2}, \ldots$ having cumulative distribution function $H_{2}$ and being independent on the renewal process $\left\{N_{2}(t), t \geq 0\right\}$. Let $\tilde{T}_{i}$ denote the

*Corresponding author. Email: krzysztof.szajowski@pwr.wroc.pl

†Email: anna.karpowicz@pwr.wroc.pl 
moment of the $i$ th claim after the time of change $s\left(\tilde{T}_{0}=s\right)$, then like before, the random variables $\tilde{S}_{i}=\tilde{T}_{i}-\tilde{T}_{i-1}$ are i.i.d. with continuous cumulative distribution function $F_{2}$.

Let $U(s, t)$ correspond to the value of the capital assets at time $t$, if the change of the parameters took place at time $s$, then

$$
U(s, t)= \begin{cases}a+c_{1} t-\sum_{n=0}^{N_{1}(t)} X_{n} & \text { if } t \leq s \\ a+c_{1} s-\sum_{n=0}^{N_{1}(s)} X_{n}+c_{2}(t-s)-\sum_{n=0}^{N_{2}(t-s)} \tilde{X}_{n} & \text { if } t>s,\end{cases}
$$

where $X_{0}=0, \tilde{X}_{0}=0$. It is convenient to introduce classical Cramér-Lunberg risk process

$$
U_{1}(t)=a+c_{1} t-\sum_{n=0}^{N_{1}(t)} X_{n}
$$

which is the base of many models widely discussed in the literature (see Azuce and Roberts [1], Muciek [13], Rolski et al. [18]). Additionally, let

$$
U_{2}(t)=c_{2} t-\sum_{n=0}^{N_{2}(t)} \tilde{X}_{n}
$$

Then, formula (1) reduces to

$$
U(s, t)= \begin{cases}U_{1}(t) & \text { if } t \leq s \\ U_{1}(s)+U_{2}(t-s) & \text { if } t>s .\end{cases}
$$

The return at time $t$ is given by the process $\{Z(s, t), t>0, s>0\}$ defined by

$$
Z(s, t)=\left\{\begin{array}{ll}
g(U(s, t)) I_{\{U(s, l)>0, l \leq t\}} & \text { if } t \leq t_{0} \\
0 & \text { if } t>t_{0}
\end{array},\right.
$$

where $t_{0}$ is a fixed horizon and $g$ is an utility function.

Assumption 1 . The utility function $g$ is bounded, continuous, nondecreasing and differentiable.

The above assumptions are not particularly restrictive, because most frequently used utility functions fulfill these requirements. The return is equal to zero only if the company goes bankrupt (i.e. $U(s, t) \leq 0$ occurs for some time $t \leq t_{0}$ ) or the horizon $t_{0}$ is exceeded.

For simplicity, we define $g(u, t)=g(u) I_{\{t \geq 0\}}$ and

$$
\begin{aligned}
\mu_{n} & =\prod_{j=1}^{n} I_{\left\{U\left(s, T_{j}\right)>0, T_{j} \leq s\right\}}=\prod_{j=1}^{n} I_{\left\{U_{1}\left(T_{j}\right)>0\right\}}, \quad \mu_{0}=1, \\
\mu_{s, n} & =\prod_{j=1}^{n} I_{\left\{U\left(s, \tilde{T}_{j}\right)>0, \tilde{T}_{j}>s\right\}}, \quad \mu_{s, 0}=1 .
\end{aligned}
$$

The process of capital assets $U(s, t)$ decreases only at the times when successive claims occur, so we can transform equation (5) into

$$
\begin{aligned}
Z(s, t) & =g\left(U(s, t), t_{0}-t\right) \prod_{j=1}^{N_{1}(s \wedge t)} I_{\left\{U\left(s, T_{j}\right)>0\right\}} \prod_{j=1}^{N_{2}\left((t-s)^{+}\right)} I_{\left\{U\left(s, \tilde{T}_{j}\right)>0\right\}} \\
& =g\left(U(s, t), t_{0}-t\right) \mu_{N_{1}(s \wedge t)} \mu_{s, N_{2}\left((t-s)^{+}\right) .}
\end{aligned}
$$


The process $Z(s, t)$ is piecewise-deterministic and belongs to the class of semi-Markov processes. The optimal stopping of such processes was studied in a fairly general setting by Boshuizen and Gouwleeuw [2], Jensen [11] and Schöttl [19].

\section{The optimization problem}

The multiple optimal stopping problems have been treated by many authors. The double stopping problem was posed by Haggstrom [10] and for the discrete time Markov processes has been considered by Eidukjavicjus [6], M.L. Nikolaev [16,17] and Stadje [20].

Let us define $\sigma$-field generated by all events up to time $t$, if there was no change of parameters

$$
\mathcal{F}_{t}=\sigma\left(U_{1}(l), l \leq t \leq s\right)=\sigma\left(X_{1}, T_{1}, \ldots, X_{N_{1}(t)}, T_{N_{1}(t)}\right)
$$

and similarly $\sigma$-field generated by all events up to time $t$ if there was change of parameters at time $s$

$$
\mathcal{F}_{s, t}=\sigma(U(s, l), l \leq t, s \leq t)=\sigma\left(\mathcal{F}_{s}, \tilde{X}_{1}, \tilde{T}_{1}, \ldots, \tilde{X}_{N_{2}(t-s)}, \tilde{T}_{N_{2}(t-s)}\right) .
$$

Additionally, we denote $\mathcal{F}_{n}:=\mathcal{F}_{T_{n}}, \mathcal{F}_{s, n}:=\mathcal{F}_{s, \tilde{T}_{n}}$ and notice that $\mathcal{F}_{s, s}=\mathcal{F}_{s}$. Let $\mathcal{T}$ and $\mathcal{T}^{s}$ be the sets of stopping times with respect to the $\sigma$-fields $\left\{\mathcal{F}_{t}, t \geq 0\right\}$ and $\left\{\mathcal{F}_{s, t}, 0 \leq s \leq t\right\}$, respectively. Furthermore, let

$$
\mathcal{T}_{n, K}=\left\{\tau \in \mathcal{T}: \tau \geq 0, T_{n} \leq \tau \leq T_{K}\right\}, \quad \text { for } n \in \mathbb{N} \text { and } n<K,
$$

and

$$
\mathcal{T}_{n, K}^{s}=\left\{\tau \in \mathcal{T}^{s}: \tau \geq s, \tilde{T}_{n} \leq \tau \leq \tilde{T}_{K}\right\}, \quad \text { for } n \in \mathbb{N} \text { and } n<K .
$$

Our goal is to find optimal stopping times $\tau^{*}$ and $\tilde{\tau}^{*}$ such that

$$
E Z\left(\tau^{*}, \tilde{\tau}^{*}\right)=\sup _{\tau \in \mathcal{T}} \sup _{\tilde{\tau} \in \mathcal{T}^{\tau}} E Z(\tau, \tilde{\tau}) .
$$

Let us notice that

$$
\begin{aligned}
E Z\left(\tau^{*}, \tilde{\tau}^{*}\right) & =\sup _{\tau \in \mathcal{T}} E Z\left(\tau, \tilde{\tau}^{*}\right)=\sup _{\tau \in \mathcal{T}} E\left\{E\left[Z\left(\tau, \tilde{\tau}^{*}\right) \mid \mathcal{F}_{\tau}\right]\right\} \\
& =\sup _{\tau \in \mathcal{T}} E \underset{\tau \in \mathcal{T}^{\tau}}{\operatorname{ess} \sup } E\left[Z(\tau, \tilde{\tau}) \mid \mathcal{F}_{\tau}\right]=\sup _{\tau \in \mathcal{T}} E J(\tau),
\end{aligned}
$$

where

$$
J(s)=E\left\{Z\left(s, \tilde{\tau}^{*}\right) \mid \mathcal{F}_{s}\right\}=\underset{\tilde{\tau} \in \mathcal{T}^{s}}{\operatorname{ess} \sup } E\left\{Z(s, \tilde{\tau}) \mid \mathcal{F}_{s}\right\} .
$$

Therefore, in order to find $\tau^{*}$ and $\tilde{\tau}^{*}$, we first calculate the process $J(s), s \geq 0$, which is revenue function in the one stopping problem if the process starts at the moment $s$. A similar problem for the classical risk process (2) has been considered by Ferenstein and Sierociński [8] and we will take the advantage of their solution. First, they have found the optimal stopping rule in the finite horizon case (i.e. assuming that at least $K$ claims occurred, where $K$ is fixed). Afterwards, they have proved that the optimal 
stopping time for the infinite horizon case is the limit of the finite horizon optimal stopping rules.

\section{Construction of the optimal second stopping time}

\subsection{Fixed number of claims}

In this section, we find the solution of one stopping problem defined by equation (9) in the case with a fixed number of claims $K$ after the change of parameters. In other words, we are looking for optimal stopping time in the class $\mathcal{T}_{0, K}^{s}$, i.e. stopping time $\tilde{\tau}_{K}^{*}:=\tilde{\tau}_{0, K}^{*}$ such that

$$
E\left\{Z\left(s, \tilde{\tau}_{K}^{*}\right) \mid \mathcal{F}_{s}\right\}=\sup _{\tilde{\tau} \in \mathcal{T}_{0, K}^{s}} E\left\{Z(s, \tilde{\tau}) \mid \mathcal{F}_{s}\right\}
$$

where $s \geq 0$ is a fixed time of the change of the parameters.

Let us define

$$
\Gamma_{n, K}^{s}=E\left\{Z\left(s, \tilde{\tau}_{n, K}^{*}\right) \mid \mathcal{F}_{s}\right\}=\underset{\tilde{\tau} \in \mathcal{T}_{n, K}^{s}}{\operatorname{ess} \sup } E\left(Z(s, \tilde{\tau}) \mid \mathcal{F}_{s, n}\right), \quad n=K, K-1, \ldots, 1,
$$

and notice that $\Gamma_{K, K}^{s}=Z\left(s, \tilde{T}_{K}\right)=g\left(U\left(s, \tilde{T}_{K}\right), t_{0}-\tilde{T}_{K}\right) \mu_{N_{1}(s)} \mu_{s, K}$. The following lemma plays a crucial role in our subsequent consideration (see Ref. [3]).

Lemma 3.1. If $\tau \in \mathcal{T}_{n, K}$ (or $\tilde{\tau} \in \mathcal{T}_{n, K}^{s}$ ), then there exists a positive, $\mathcal{F}_{n^{-}}$-measurable (or $\mathcal{F}_{s, n^{-}}$ measurable), random variable $R_{n}$ (or $\tilde{R}_{n}$ ) such that

$$
\tau \wedge T_{n+1}=\left(T_{n}+R_{n}\right) \wedge T_{n+1}, \quad\left(\text { or } \tilde{\tau} \wedge \tilde{T}_{n+1}=\left(\tilde{T}_{n}+\tilde{R}_{n}\right) \wedge \tilde{T}_{n+1}\right) \text { a.s. }
$$

We derive the dynamic programming equations satisfied by $\Gamma_{n, K}^{s}$ in a similar way to Ferenstein and Sierociński [8].

THEOREM 3.1. Let $s \geq 0$ be the time of change of the parameters.

(i) For $n=K-1, K-2, \ldots, 0$

$$
\begin{aligned}
\Gamma_{n, K}^{s}= & \operatorname{ess} \sup \left\{\mu_{N_{1}(s)} \mu_{s, n} \bar{F}_{2}\left(\tilde{R}_{n}\right) g\left(U\left(s, \tilde{T}_{n}\right)+c_{2} \tilde{R}_{n}, t_{0}-\tilde{T}_{n}-\tilde{R}_{n}\right)\right. \\
& \left.+E\left[I_{\left\{\tilde{R}_{n} \geq \tilde{S}_{n+1}\right\}} \Gamma_{n+1, K}^{s} \mid \mathcal{F}_{s, n}\right]: \tilde{R}_{n} \geq 0 \text { is } \mathcal{F}_{s, n}-\text { measurable }\right\} \text { a.s. }
\end{aligned}
$$

where $\bar{F}_{2}=1-F_{2}$ is the survival function.

(ii) Denote

$$
\Gamma_{n, K}^{s}=\mu_{N_{1}(s)} \mu_{s, n} \tilde{\gamma}_{K-n}\left(U\left(s, \tilde{T}_{n}\right), \tilde{T}_{n}\right) \text { a.s., }
$$

for $n=K, K-1, \ldots, 0$, where the sequence of functions $\tilde{\gamma}_{j}(u, t), u \in \mathbb{R}, t>s$, is defined recursively as follows:

$$
\begin{aligned}
\tilde{\gamma}_{0}(u, t)= & g\left(u, t_{0}-t\right) \\
\tilde{\gamma}_{j}(u, t)= & \sup _{r \geq 0}\left[\bar{F}_{2}(r) g\left(u+c_{2} r, t_{0}-t-r\right)\right. \\
& \left.+\int_{0}^{r} \mathrm{~d} F_{2}(z) \int_{0}^{u+c_{2} z} \tilde{\gamma}_{j-1}\left(u+c_{2} z-x, t+z\right) \mathrm{d} H_{2}(x)\right], \quad j=1,2, \ldots
\end{aligned}
$$


Proof.

(i) Let us take $\tilde{\tau} \in \mathcal{T}_{n, K}^{s}$. From Lemma 3.1, we get

$$
\begin{aligned}
& A_{n}=\left\{\tilde{\tau}<\tilde{T}_{n+1}\right\}=\left\{\tilde{T}_{n}+\tilde{R}_{n}<\tilde{T}_{n+1}\right\}=\left\{\tilde{R}_{n}<\tilde{S}_{n+1}\right\}, \\
& \bar{A}_{n}=\left\{\tilde{\tau} \geq \tilde{T}_{n+1}\right\}=\left\{\tilde{T}_{n}+\tilde{R}_{n} \geq \tilde{T}_{n+1}\right\}=\left\{\tilde{R}_{n} \geq \tilde{S}_{n+1}\right\},
\end{aligned}
$$

then $E\left\{Z(s, \tilde{\tau}) \mid \mathcal{F}_{s, n}\right\}=E\left\{Z(s, \tilde{\tau}) I_{A_{n}} \mid \mathcal{F}_{s, n}\right\}+E\left\{Z(s, \tilde{\tau}) I_{\bar{A}_{n}} \mid \mathcal{F}_{s, n}\right\}=\alpha_{n}+\beta_{n}$.

Let us notice, on the basis of Lemma 3.1, that for $\tilde{\tau}<\tilde{T}_{n+1}$ occurs $\tilde{\tau}=\tilde{T}_{n}+\tilde{R}_{n}$ a.s. and it follows $U(s, \tilde{\tau})=U\left(s, \tilde{T}_{n}\right)+c_{2} \tilde{R}_{n}$ a.s. Now we calculate $\alpha_{n}$ and $\beta_{n}$

$$
\begin{aligned}
\alpha_{n} & =E\left\{\mu_{N_{1}(s \wedge \tilde{\tau})} \mu_{s, N_{2}\left((\tilde{\tau}-s)^{+}\right)} g\left(U(s, \tilde{\tau}), t_{0}-\tilde{\tau}\right) I_{A_{n}} \mid \mathcal{F}_{s, n}\right\} \\
& =E\left\{\mu_{N_{1}(s)} \mu_{s, n} g\left(U\left(s, \tilde{T}_{n}\right)+c_{2} \tilde{R}_{n}, t_{0}-\tilde{T}_{n}-\tilde{R}_{n}\right) I_{\left\{\tilde{R}_{n}<\tilde{S}_{n+1}\right\}} \mid \mathcal{F}_{s, n}\right\} \\
& =\mu_{N_{1}(s)} \mu_{s, n} \bar{F}_{2}\left(\tilde{R}_{n}\right) g\left(U\left(s, \tilde{T}_{n}\right)+c_{2} \tilde{R}_{n}, t_{0}-\tilde{T}_{n}-\tilde{R}_{n}\right) .
\end{aligned}
$$

Let $\tilde{\tau}^{\prime}=\tilde{\tau} \vee \tilde{T}_{n+1} \in \mathcal{T}_{n+1, K}^{s}$, hence we obtain

$$
\beta_{n}=E\left\{I_{\left\{\tilde{R}_{n} \geq \tilde{S}_{n+1}\right\}} E\left(Z\left(s, \tilde{\tau}^{\prime}\right) \mid \mathcal{F}_{s, n+1}\right) \mid \mathcal{F}_{s, n}\right\}
$$

We get the dynamic programming equation for $\Gamma_{n, K}^{s}$ using the standard argumentation for optimal stopping theory.

(ii) Now we proceed by backward induction. First we check that (ii) is satisfied for $n=K$

$$
\Gamma_{K, K}^{s}=\mu_{N_{1}(s)} \mu_{s, K} \tilde{\gamma}_{0}\left(U\left(s, \tilde{T}_{K}\right), \tilde{T}_{K}\right)=\mu_{N_{1}(s)} \mu_{s, K} g\left(U\left(s, \tilde{T}_{K}\right), t_{0}-\tilde{T}_{K}\right) .
$$

Let $n=K-1$ then from (i), we get

$$
\begin{aligned}
\Gamma_{K-1, K}^{s}= & \operatorname{ess} \sup \left\{\mu_{N_{1}(s)} \mu_{s, K-1} \bar{F}_{2}\left(\tilde{R}_{K-1}\right) g\left(U\left(s, \tilde{T}_{K-1}\right)+c_{2} \tilde{R}_{K-1}, t_{0}-\tilde{T}_{K-1}-\tilde{R}_{K-1}\right)\right. \\
& +E\left[I_{\left\{\tilde{R}_{K-1} \geq \tilde{S}_{K}\right\}} \mu_{N_{1}(s)} \mu_{s, K} \tilde{\gamma}_{0}\left(U\left(s, \tilde{T}_{K}\right), \tilde{T}_{K}\right) \mid \mathcal{F}_{s, K-1}\right] \\
& \left.: \tilde{R}_{K-1} \geq 0 \text { is } \mathcal{F}_{s, K-1}-\text { measurable }\right\} \text { a.s. }
\end{aligned}
$$

It is easily seen that $\mu_{s, K}=\mu_{s, K-1} I_{\left\{U\left(s, \tilde{T}_{K-1}\right)+c_{2} \tilde{S}_{K}-\tilde{X}_{K}>0\right\}}$ and random variables $\tilde{S}_{K}, \tilde{X}_{K}$ are independent on the $\sigma$-field $\mathcal{F}_{s, K-1}$. An easy computation shows that

$$
\Gamma_{K-1, K}^{s}=\mu_{N_{1}(s)} \mu_{s, K-1} \tilde{\gamma}_{1}\left(U\left(s, \tilde{T}_{K-1}\right), \tilde{T}_{K-1}\right) .
$$

Let $1 \leq n \leq K-1$ and suppose $\Gamma_{n, K}^{s}=\mu_{N_{1}(s)} \mu_{s, n} \tilde{\gamma}_{K-n}\left(U\left(s, \tilde{T}_{n}\right), \tilde{T}_{n}\right)$.

We apply (i)

$$
\begin{aligned}
\Gamma_{n-1, K}^{s}= & \operatorname{ess} \sup \left\{\mu_{N_{1}(s)} \mu_{s, n-1} \bar{F}_{2}\left(\tilde{R}_{n-1}\right) g\left(U\left(s, \tilde{T}_{n-1}\right)+c_{2} \tilde{R}_{n-1}, t_{0}-\tilde{T}_{n-1}-\tilde{R}_{n-1}\right)\right. \\
& +E\left[I_{\left\{\tilde{R}_{n-1} \geq \tilde{S}_{n}\right\}} \mu_{N_{1}(s)} \mu_{s, n} \tilde{\gamma}_{K-n}\left(U\left(s, \tilde{T}_{n}\right), \tilde{T}_{n}\right) \mid \mathcal{F}_{s, n-1}\right] \\
& \left.: \tilde{R}_{n-1} \geq 0 \text { is } \mathcal{F}_{s, n-1}-\text { measurable }\right\} \text { a.s. }
\end{aligned}
$$

and express the second term under ess sup as

$\mu_{N_{1}(s)} \mu_{s, n-1} E\left[I_{\left\{\tilde{R}_{n-1} \geq \tilde{S}_{n}\right\}} I_{\left\{U\left(s, \tilde{T}_{n-1}\right)+c_{2} \tilde{S}_{n}-\tilde{X}_{n}>0\right\}} \tilde{\gamma}_{K-n}\left(U\left(s, \tilde{T}_{n-1}\right)+c_{2} \tilde{S}_{n}-\tilde{X}_{n}, \tilde{T}_{n-1}+\tilde{S}_{n}\right) \mid \mathcal{F}_{s, n-1}\right]$. 
We see at once that

$$
\begin{aligned}
\Gamma_{n-1, K}^{s}= & \mu_{N_{1}(s)} \mu_{s, n-1} \operatorname{ess} \sup \left\{\bar{F}_{2}\left(\tilde{R}_{n-1}\right) g\left(U\left(s, \tilde{T}_{n-1}\right)+c_{2} \tilde{R}_{n-1}, t_{0}-\tilde{T}_{n-1}-\tilde{R}_{n-1}\right)\right. \\
& +\int_{0}^{\tilde{R}_{n-1}} \mathrm{~d} F_{2}(z) \int_{0}^{U\left(s, \tilde{T}_{n-1}\right)+c_{2} z} \tilde{\gamma}_{K-n}\left(U\left(s, \tilde{T}_{n-1}\right)+c_{2} z-x, \tilde{T}_{n-1}+z\right) \mathrm{d} H_{2}(x) \\
& \left.: \tilde{R}_{n-1} \geq 0 \text { is } \mathcal{F}_{s, n}-\text { measurable }\right\}=\mu_{N_{1}(s)} \mu_{s, n-1} \tilde{\gamma}_{K-(n-1)}\left(U\left(s, \tilde{T}_{n-1}\right), \tilde{T}_{n-1}\right) \text { a.s. }
\end{aligned}
$$

In order to perform $\tilde{\tau}_{K}^{*}$, the properties of the sequence $\left\{\tilde{\gamma}_{j}, j \geq 0\right\}$ are presented according to the idea in Ref. [8]. Let $B=B[(-\infty, \infty) \times[0, \infty)]$ be the space of all bounded continuous functions with the norm $\|\delta\|=\sup _{u, t}|\delta(u, t)|$ and

$$
B^{0}=\left\{\delta: \delta(u, t)=\delta_{1}(u, t) I_{\left\{t \leq t_{0}\right\}} \text { and } \delta_{1} \in B\right\} .
$$

Let us define, for each $\delta \in B^{0}, u \in \mathbb{R}, t>s, r \in \mathbb{R}^{+}$, the function $\tilde{\phi}_{\delta}$

$$
\tilde{\phi}_{\delta}(r, u, t)=\bar{F}_{2}(r) g\left(u+c_{2} r, t_{0}-t-r\right)+\int_{0}^{r} \mathrm{~d} F_{2}(z) \int_{0}^{u+c_{2} z} \delta\left(u+c_{2} z-x, t+z\right) \mathrm{d} H_{2}(x)
$$

and the operator $\tilde{\Phi}$

$$
(\tilde{\Phi} \delta)(u, t)=\sup _{r \geq 0}\left\{\tilde{\phi}_{\delta}(r, u, t)\right\} \in B^{0} .
$$

Note that $\tilde{\phi}_{\delta}(r, u, t)$ is continuous and differentiable with respect to $r, u, t$, for $t \in\left[0, t_{0}-r\right]$. It follows from the properties of the cumulative distribution function $F_{2}$ and utility function $g$.

Lemma 3.2. For each $\delta \in B^{0}$ we have

$$
(\tilde{\Phi} \delta)(u, t)=\max _{0 \leq r \leq t_{0}-t}\left\{\tilde{\phi}_{\delta}(r, u, t)\right\} \in B^{0}
$$

and there exists a function $\tilde{r}_{\delta}(u, t)$ such that $(\tilde{\Phi} \delta)(u, t)=\tilde{\phi}_{\delta}\left(\tilde{r}_{\delta}(u, t), u, t\right)$.

Proof. It is easy to check that

$$
\tilde{\phi}_{\delta}(r, u, t)=\int_{0}^{t_{0}-t} \mathrm{~d} F_{2}(z) \int_{0}^{u+c_{2} z} \delta\left(u+c_{2} z-x, t+z\right) \mathrm{d} H_{2}(x)
$$

for all $\delta \in B^{0}$ and for any $r>t_{0}-t$. The form of $\tilde{\Phi}$ is a straightforward consequence of Assumption 1 and the fact that $F_{2}$ is continuous.

Let us perform recursive formula for $\tilde{\gamma}_{j}(u, t)$

$$
\tilde{\gamma}_{j}(u, t)= \begin{cases}\left(\tilde{\Phi} \tilde{\gamma}_{j-1}\right)(u, t) & \text { if } u \geq 0 \text { and } s<t \leq t_{0}, \\ 0 & \text { otherwise. }\end{cases}
$$

By Lemma 3.2, there exists function $\tilde{r}_{j}:=\tilde{r}_{\tilde{\gamma}_{j-1}}$ such that

$$
\tilde{\gamma}_{j}(u, t)= \begin{cases}\tilde{\phi} \tilde{\gamma}_{j-1}\left(\tilde{r}_{j}(u, t), u, t\right) & \text { if } u \geq 0 \text { and } s<t \leq t_{0} \\ 0 & \text { otherwise. }\end{cases}
$$

The consequence of the foregoing equations is the theorem, which determines optimal stopping times $\tilde{\tau}_{n, K}^{*}$ in following manner: 
THEOREM 3.2. The stopping time $\tilde{\tau}_{n, K}^{*}=\tilde{T}_{\tilde{\eta}_{n, K}}+\tilde{R}_{\tilde{\eta}_{n, K}}^{*}, n=0,1, \ldots, K$, where

$$
\tilde{R}_{i}^{*}=\tilde{r}_{K-i}\left(U\left(s, \tilde{T}_{i}\right), \tilde{T}_{i}\right) \quad \tilde{\eta}_{n, K}=K \wedge \inf \left\{i \geq n: \tilde{R}_{j}^{*}<\tilde{S}_{i+1}\right\},
$$

is optimal in the class $\mathcal{T}_{n, K}^{s}$.

For this $\tilde{\tau}_{n, K}^{*}$, we have $\Gamma_{n, K}^{s}=E\left(Z\left(s, \tilde{\tau}_{n, K}^{*}\right) \mid \mathcal{F}_{s, n}\right)$ and for $n=0$, we have $\Gamma_{0, K}^{s}=$ $E\left(Z\left(s, \tilde{\tau}_{K}^{*}\right) \mid \mathcal{F}_{s}\right)=\mu_{N_{1}(s)} \tilde{\gamma}_{K}\left(U_{1}(s), s\right)$.

\subsection{Infinite number of claims}

In this section, we consider the case of infinite number of claims and we find stopping time $\tilde{\tau}^{*}$, which is optimal in the class $\mathcal{T}^{s}$. In order to solve our one stopping problem, it is necessary to put the following restriction on cumulative distribution function.

Assumption 2. $F_{2}\left(t_{0}\right)<1$.

The following lemma (see Ref. [8]) will play the important role in our considerations.

Lemma 3.3. The operator $\tilde{\Phi}: B^{0} \rightarrow B^{0}$ defined by equation (11) is a contraction.

Proof. Let us take the functions $\delta_{1}, \delta_{2} \in B^{0}$. On the basis of Lemma 3.2, there exist $\tilde{\rho}_{i}=\tilde{r}_{\delta_{i}}(u, t)$, such that $\left(\tilde{\Phi} \delta_{i}\right)(u, t)=\tilde{\phi}_{\delta_{i}}\left(\tilde{\rho}_{i}, u, t\right), i=1,2$. Let us notice that $\tilde{\phi}_{\delta_{2}}\left(\tilde{\rho}_{2}, u, t\right) \geq$ $\tilde{\phi}_{\delta_{2}}\left(\tilde{\rho}_{1}, u, t\right)$ and it follows that

$$
\begin{aligned}
\left(\tilde{\Phi} \delta_{1}\right)(u, t)-\left(\tilde{\Phi} \delta_{2}\right)(u, t)= & \tilde{\phi}_{\delta_{1}}\left(\tilde{\rho}_{1}, u, t\right)-\tilde{\phi}_{\delta_{2}}\left(\tilde{\rho}_{2}, u, t\right) \leq \tilde{\phi}_{\delta_{1}}\left(\tilde{\rho}_{1}, u, t\right)-\tilde{\phi}_{\delta_{2}}\left(\tilde{\rho}_{1}, u, t\right) \\
& \leq \int_{0}^{\tilde{\rho_{1}}} \mathrm{~d} F_{2}(z) \int_{0}^{u+c_{2} z}\left[\delta_{1}-\delta_{2}\right]\left(u+c_{2} z-x, t+z\right) \mathrm{d} H_{2}(x) \\
& \leq\left\|\delta_{1}-\delta_{2}\right\| \int_{0}^{\tilde{\rho_{1}}} \mathrm{~d} F_{2}(z) \int_{0}^{u+c_{2} z} \mathrm{~d} H_{2}(x) \leq \tilde{\rho}\left\|\delta_{1}-\delta_{2}\right\|,
\end{aligned}
$$

where

$$
\tilde{\rho}=\sup _{u>0} \int_{0}^{t_{0}} \mathrm{~d} F_{2}(z) \int_{0}^{u+c_{2} z} \mathrm{~d} H_{2}(x) \leq F_{2}\left(t_{0}\right)<1 .
$$

Similarly, we get $\left(\tilde{\Phi} \delta_{2}\right)(u, t)-\left(\tilde{\Phi} \delta_{1}\right)(u, t) \leq \tilde{\rho}\left\|\delta_{1}-\delta_{2}\right\|$. Hence, $\left\|\tilde{\Phi} \delta_{1}-\tilde{\Phi} \delta_{2}\right\| \leq$ $\tilde{\rho}\left\|\delta_{1}-\delta_{2}\right\|$.

As $\tilde{\gamma}_{0} \in B^{0}$, we conclude that $\tilde{\gamma}_{i} \in B^{0}$ for all $i$, therefore we can use the fixed point theorem and obtain the following lemma

Lemma 3.4. There exists $\tilde{\gamma} \in B^{0}$ such that

$$
\tilde{\gamma}=\tilde{\Phi} \tilde{\gamma} \quad \text { and } \quad \lim _{K \rightarrow \infty}\left\|\tilde{\gamma}_{K}-\tilde{\gamma}\right\|=0 .
$$

COROLlary 3.3. $\tilde{\gamma}$ is uniform limit of $\tilde{\gamma}_{K}$, when $K$ tends to infinity.

The consideration of Sections 3.1 and 3.2 leads to the following formulation of the optimal strategy after the change of parameters in the risk process. 
TheOrem 3.4. If the function $g$ fulfils Assumption $1, F_{2}$ fulfils Assumption 2 and has the density function $f_{2}$, then

(i) for $n \in \mathbb{N}$, the limit $\tilde{\tau}_{n}^{*}=\lim _{K \rightarrow \infty} \tilde{\tau}_{n, K}^{*}$ a.s. exists and $\tilde{\tau}_{n}^{*}$ is an optimal stopping rule in the set $\mathcal{T}^{s} \cap\left\{\tilde{\tau} \geq \tilde{T}_{n}\right\}$,

(ii) $E\left(Z\left(s, \tilde{\tau}_{n}^{*}\right) \mid \mathcal{F}_{s, n}\right)=\mu_{N_{1}(s)} \mu_{s, n} \tilde{\gamma}\left(U\left(s, \tilde{T}_{n}\right), \tilde{T}_{n}\right)$ a.s.

Proof.

(i) The stopping rule $\tilde{\tau}_{n}^{*}=\lim _{K \rightarrow \infty} \tilde{\tau}_{n, K}^{*}$ a.s. exists because $\tilde{\tau}_{n, K}^{*} \leq \tau_{n, K+1}^{*}$.

Let us define the process $\xi(s, t)=(t, U(s, t), Y(s, t), V(s, t))$, where $Y(s, t)=t-$ $\tilde{T}_{N_{2}\left((t-s)^{+}\right)}, V(s, t)=\mu_{N_{1}(s)} \mu_{s, N_{2}\left((t-s)^{+}\right)}$. It can be found in Gikhman and Skorohod [9] that $\xi(s, t)$ is a Markov process with the state space $\mathbb{R}^{+} \times \mathbb{R} \times \mathbb{R}^{+} \times\{0,1\}$. We express the process $Z(s, t)$ as $Z(s, t)=\tilde{g}(\xi(s, t))$ and calculate the strong generator of $\xi(s, t)$

$$
(A \tilde{g})(t, u, y, v)=v\left\{c_{2} g^{\prime}(u)+\frac{f_{2}(y)}{\bar{F}_{2}(y)}\left[\int_{0}^{u} g(u-x) \mathrm{d} H_{2}(x)-g(u)\right]\right\},
$$

where $s<t<t_{0}, y>0$ and $v \in\{0,1\}$.

The next step is noting that $\tilde{g}(\xi(s, t))-\tilde{g}(\xi(s, s))-\int_{s}^{t}(A \tilde{g})(\xi(s, z)) \mathrm{d} z$ is a martingale with respect to $\sigma(\xi(s, z), s<z \leq t)$, which is the same as $\mathcal{F}_{s, t}$ (see Davis [5]) and applying the optional sampling theorem

$$
E\left\{\tilde{g}\left(\xi\left(s, \tilde{\tau}_{n, K}^{*}\right)\right) \mid \mathcal{F}_{s, n}\right\}-\tilde{g}\left(\xi\left(s, \tilde{T}_{n}\right)\right)=E\left[\int_{\tilde{T}_{n}}^{\tilde{\tau}_{n, K}^{*}}(A \tilde{g})(\xi(s, z)) \mathrm{d} z \mid \mathcal{F}_{s, n}\right] \text { a.s. }
$$

It is immediate that

$$
\begin{aligned}
(A \tilde{g})(\xi(s, t))= & \mu_{N_{1}(s)} \mu_{N_{2}(t-s)}\left\{c_{2} g^{\prime}(U(s, t))\right. \\
& \left.+\frac{f_{2}\left(t-\tilde{T}_{N_{2}(t-s)}\right)}{\bar{F}_{2}\left(t-\tilde{T}_{N_{2}(t-s)}\right)}\left[\int_{0}^{U(s, t)} g(U(s, t)-x)\right) \mathrm{d} H_{2}(x)-g(U(s, t)]\right\},
\end{aligned}
$$

therefore the right side of equation (12) can be expressed as $E\left\{I_{n, K}^{1} \mid \mathcal{F}_{s, n}\right\}-$ $E\left\{I_{n, K}^{2} \mid \mathcal{F}_{s, n}\right\}$, where

$$
\left.I_{n, K}^{2}=\int_{\tilde{T}_{n}}^{\tilde{\tau}_{n, K}^{*}} \mu_{N_{1}(s)} \mu_{N_{2}(z-s)} \frac{f_{2}\left(z-\tilde{T}_{N_{2}(z-s)}\right)}{\bar{F}_{2}\left(z-\tilde{T}_{N_{2}(z-s)}\right)} g(U(s, z)) \mathrm{d} z\right\} .
$$

We can observe that $I_{n, K}^{1}, I_{n, K}^{2}$ are positive random variables. Let us define random variable $M=\inf \left\{n \in \mathbb{N}: \tilde{T}_{n}<t_{0}, \tilde{T}_{n+1} \geq t_{0}\right\}$, then

$$
I_{n, K}^{2} \leq \frac{g\left(a+c_{2}\left(t_{0}-s\right)\right)}{\bar{F}_{2}\left(t_{0}\right)} E M \text { a.s., }
$$

where $E M=\sum_{n=0}^{\infty} F_{2}^{*(n)}\left(t_{0}\right) \leq \sum_{n=0}^{\infty}\left[F_{2}\left(t_{0}\right)\right]^{n}<\infty$. On the basis of monotone convergence theorem, we see that

$$
E\left[\int_{\tilde{T}_{n}}^{\tilde{\tau}_{n, K}^{*}}(A \tilde{g})(\xi(s, z)) \mathrm{d} z \mid \mathcal{F}_{s, n}\right] \underset{K \rightarrow \infty}{\longrightarrow} E\left[\int_{\tilde{T}_{n}}^{\tilde{\tau}_{n}^{*}}(A \tilde{g})(\xi(s, z)) \mathrm{d} z \mid \mathcal{F}_{s, n}\right] \text { a.s. }
$$


Applying the Dynkin's formula again yields

$$
E\left[\int_{\tilde{T}_{n}}^{\tilde{\tau}_{n}^{*}}(A \tilde{g})(\xi(s, z)) \mathrm{d} z \mid \mathcal{F}_{s, n}\right]=E\left\{\tilde{g}\left(\xi\left(s, \tilde{\tau}_{n}^{*}\right)\right) \mid \mathcal{F}_{s, n}\right\}-\tilde{g}\left(\xi\left(s, \tilde{T}_{n}\right)\right) \text { a.s. }
$$

Combining these with equations (12) and (13), we obtain

$$
E\left\{\tilde{g}\left(\xi\left(s, \tilde{\tau}_{n, K}^{*}\right)\right) \mid \mathcal{F}_{s, n}\right\} \stackrel{K \rightarrow \infty}{\longrightarrow} E\left\{\tilde{g}\left(\xi\left(s, \tilde{\tau}_{n}^{*}\right)\right) \mid \mathcal{F}_{s, n}\right\} \text { a.s. }
$$

The task is now to prove that $\tilde{\tau}_{n}^{*}$ is optimal in the $\mathcal{T}^{s} \cap\left\{\tau: \tau \geq T_{n}\right\}$. Let $\tau$ be any stopping rule $\tau \in \mathcal{T}^{s} \cap\left\{\tau: \tau \geq T_{n}\right\}$, then from optimality of $\tilde{\tau}_{n, K}^{*}$, we get

$$
E\left\{\tilde{g}\left(\xi\left(s, \tilde{\tau}_{n, K}^{*}\right)\right) \mid \mathcal{F}_{s, n}\right\} \geq E\left\{\tilde{g}\left(\xi\left(s, \tau \wedge \tilde{T}_{K}\right)\right) \mid \mathcal{F}_{s, n}\right\} \text { a.s. }
$$

In the same manner as before, we can see that $E\left\{\tilde{g}\left(\xi\left(s, \tilde{\tau}_{n}^{*}\right)\right) \mid \mathcal{F}_{s, n}\right\} \geq E\left\{\tilde{g}(\xi(s, \tau)) \mid \mathcal{F}_{s, n}\right\}$, which completes the proof.

(ii) Applying Theorem 3.1 and equation (10), we deduce that

$$
E\left\{\tilde{g}\left(\xi\left(s, \tilde{\tau}_{n, K}^{*}\right)\right) \mid \mathcal{F}_{s, n}\right\}=E\left\{Z\left(s, \tilde{\tau}_{n, K}^{*}\right) \mid \mathcal{F}_{s, n}\right\}=\mu_{N_{1}(s)} \mu_{s, n} \tilde{\gamma}_{K-n}\left(U\left(s, \tilde{T}_{n}\right), \tilde{T}_{n}\right) \text { a.s. }
$$

Combining Lemma 3.3 and equation (15), we obtain

$$
E\left\{\tilde{g}\left(\xi\left(s, \tau_{\sim}^{n, K}\right)\right) \mid \mathcal{F}_{s, n}\right\} \stackrel{K \rightarrow \infty}{\longrightarrow} E\left\{\tilde{g}\left(\xi\left(s, \tilde{\tau}^{*}\right)\right) \mid \mathcal{F}_{s, n}\right\}=\mu_{N_{1}(s)} \mu_{s, n} \tilde{\gamma}\left(U\left(s, \tilde{T}_{n}\right), \tilde{T}_{n}\right) \text { a.s. }
$$

At the end of this section, we notice that optimal stopping time for second stop is equal to $\tilde{\tau}^{*}=\tilde{\tau}_{0}^{*} \in \mathcal{T}^{s}$, where $\tilde{\tau}_{0}^{*}=\lim _{K \rightarrow \infty} \tilde{\tau}_{0, K}^{*}$ and the conditional value function of the optimal stopping problem after moment $s$ is given by

$$
J(s)=E\left\{Z\left(s, \tilde{\tau}_{0}^{*}\right) \mid \mathcal{F}_{s, 0}\right\}=\mu_{N_{1}(s)} \mu_{s, 0} \tilde{\gamma}\left(U\left(s, \tilde{T}_{0}\right), \tilde{T}_{0}\right)=\mu_{N_{1}(s)} \tilde{\gamma}\left(U_{1}(s), s\right) \text { a.s. }
$$

\section{Construction of the optimal first stopping time}

In this section, we formulate the solution of our problem. Properties of the function $\tilde{\gamma}(u, t)$, which was determined in Lemma 3.4, will play the crucial role.

Remark 4.1. The functions $\tilde{\gamma}_{j}(u, t)$ have finite one-sided derivatives.

The foregoing remark is the consequence of the fact, that $\tilde{\phi}_{\delta}(r, u, t)$ is continuous and differentiable with respect to $r, u, t$.

Remark 4.2. If the functions $\delta_{n}(u)$ have finite one-sided derivatives for all $u$ and $\delta$ is uniform limit of $\delta_{n}$, with respect to the norm $\|\delta\|=\sup _{u}|\delta(u)|$, then $\delta(u)$ has the same, finite onesided derivatives for all $u$.

Proof. The function $\delta_{n}$ has finite right-hand derivative in the point $u_{0}$, equal to $M<\infty$ if and only if

$$
\lim _{h \rightarrow 0^{+}} \frac{\delta_{n}\left(u_{0}+h\right)-\delta_{n}\left(u_{0}\right)}{h}=M
$$


and this is equivalent to the condition

$$
\forall_{\epsilon_{1}>0} \exists_{\delta_{\epsilon_{1}}>0} \forall_{h}\left(0<h<\delta_{\epsilon_{1}} \Rightarrow\left|\frac{\delta_{n}\left(u_{0}+h\right)-\delta_{n}\left(u_{0}\right)}{h}-M\right|<\epsilon_{1}\right) .
$$

On the other side, the sequence $\delta_{n}$ converge uniformly to the $\delta$, with respect to the norm supremum, if and only if

$$
\forall_{\epsilon_{2}>0} \exists_{n_{0}} \forall_{n \geq n_{0}} \forall_{u} \sup _{u}\left|\delta_{n}(u)-\delta_{n_{0}}(u)\right|<\epsilon_{2} .
$$

Let us take any $u_{0}$ and $n_{0}$ such, that $\epsilon_{2}=\epsilon_{1} h$. On the basis of the equations (17) and (18), we have

$$
\begin{aligned}
\left|\frac{\delta\left(u_{0}+h\right)-\delta\left(u_{0}\right)}{h}-M\right| \leq & \left|\frac{\delta\left(u_{0}+h\right)-\delta_{n_{0}}\left(u_{0}+h\right)}{h}\right|+\left|\frac{\delta_{n_{0}}\left(u_{0}+h\right)-\delta_{n_{0}}\left(u_{0}\right)}{h}-M\right| \\
& +\left|\frac{\delta_{n_{0}}\left(u_{0}\right)-\delta\left(u_{0}\right)}{h}\right|<\frac{\epsilon_{1} h}{h}+\epsilon_{1}+\frac{\epsilon_{1} h}{h}=3 \epsilon_{1}=\epsilon .
\end{aligned}
$$

We conclude that for all $\epsilon$, there exists $\delta=\delta_{\epsilon_{1}}$, such that $\delta_{+}^{\prime}\left(u_{0}\right)=M<\infty$. The proof for the left-hand derivative is similar.

LEMma 4.3. The function $\tilde{\gamma}(u, t)$ is bounded, continuous, nondecreasing and has finite onesided derivatives with respect to $u$ for all $u \in \mathbb{R}^{+}$and $t$ for all $t \in\left[0, t_{0}\right]$.

Proof. From Lemma 3.4, we get that $\tilde{\gamma}(u, t)$ is bounded and continuous. In order to prove that $\tilde{\gamma}(u, t)$ is nondecreasing with respect to $u$, we first observe that on the basis of equations (6) and (16) for $\mu_{N_{1}(s)}=1, s<\tilde{\tau}_{0}^{*} \leq t_{0}$, we have

$$
\tilde{\gamma}\left(U_{1}(s), s\right)=E\left\{\mu_{s, N_{2}\left(\tau_{0}^{*}-s\right)} g\left(U_{1}(s)+U_{2}\left(\tau_{0}^{*}-s\right)\right) \mid \mathcal{F}_{s}\right\} \text { a.s. }
$$

Let us notice that $\mathcal{F}_{s}$ can be replaced with $U_{1}(s)$ and therefore

$$
\tilde{\gamma}\left(U_{1}(s), s\right)=E\left\{\mu_{s, N_{2}\left(\tilde{\tau}_{0}^{*}-s\right)} g\left(u+U_{2}\left(\tilde{\tau}_{0}^{*}-s\right)\right) \mid U_{1}(s)=u\right\} \text { a.s. }
$$

As the function $g(u)$ is nondecreasing on the basis of Assumption 1 and $\tau_{0}^{*}$ is optimal if $U_{1}(s)=u$, we conclude that $\tilde{\gamma}(u, t)$ is nondecreasing with respect to $u$. The straightforward consequence of Remark 4.1, Corollary 3.3 and Remark 4.2 is the fact that $\tilde{\gamma}$ has finite onesided derivatives with respect to $u$ and $t$.

We have proved that the function $\tilde{\gamma}$ has similar properties as $g$ and this follows that the rest of the paper runs like in Section 3. We will apply the same arguments again, with $g$ replaced by $\tilde{\gamma}$.

\subsection{Fixed number of claims}

In this section, we find the first optimal stopping time, if the number of claims before the change of parameters is fixed and equal to $L$. We can formulate the problem as looking for $\tau_{L}^{*}=\tau_{0, L}^{*}$ such that

$$
E J\left(\tau_{L}^{*}\right)=\sup _{\tau \in \mathcal{T}_{L}} E J(\tau)
$$


Let us define

$$
\left.\Gamma_{n, L}=E\left\{J\left(\tau_{n, L}^{*}\right) \mid \mathcal{F}_{n}\right\}=\underset{\tau \in \mathcal{T}_{n, L}}{\operatorname{ess} \sup } E\left(J(\tau) \mid \mathcal{F}_{n}\right)\right), \quad n=L, L-1, \ldots, 1 .
$$

The dynamic programming equations satisfied by $\Gamma_{n, L}$ are considered in following theorem. THEOREM 4.1.

(i) For $n=L-1, L-2, \ldots, 0$

$$
\begin{aligned}
\Gamma_{n, L}= & \operatorname{ess} \sup \left\{\mu_{n} \bar{F}_{1}\left(R_{n}\right) \tilde{\gamma}\left(U_{1}\left(T_{n}\right)+c_{1} R_{n}, T_{n}+R_{n}\right)\right. \\
& \left.+E\left[I_{\left\{R_{n} \geq S_{n+1}\right\}} \Gamma_{n+1, L} \mid \mathcal{F}_{n}\right]: R_{n} \geq 0 \text { is } \mathcal{F}_{n}-\text { measurable }\right\} \text { a.s. }
\end{aligned}
$$

where $\bar{F}_{1}=1-F_{1}$ is the survival function.

(ii) The sequence of random variables $\Gamma_{n, L}$ can be performed as $\Gamma_{n, L}=\mu_{n} \gamma_{L-n}\left(U_{1}\left(T_{n}\right), T_{n}\right)$ a.s. for $n=L, L-1, \ldots, 0$, where the sequence of functions $\gamma_{j}(u, t), u \in \mathbb{R}, t \in\left[0, t_{0}\right]$ is defined recursively as follows:

$$
\begin{aligned}
\gamma_{0}(u, t)= & \tilde{\gamma}(u, t) \\
\gamma_{j}(u, t)= & \sup _{r \geq 0}\left[\bar{F}_{1}(r) \tilde{\gamma}\left(u+c_{1} r, t+r\right)\right. \\
& \left.+\int_{0}^{r} \mathrm{~d} F_{1}(z) \int_{0}^{u+c_{1} z} \gamma_{j-1}\left(u+c_{1} z-x, t+z\right) \mathrm{d} H_{1}(x)\right] \quad j=1,2, \ldots
\end{aligned}
$$

Similarly, like for one stop case, we define the function $\phi_{\delta}$

$$
\phi_{\delta}(r, u, t)=\bar{F}_{1}(r) \tilde{\gamma}\left(u+c_{1} r, t+r\right)+\int_{0}^{r} \mathrm{~d} F_{1}(z) \int_{0}^{u+c_{1} z} \delta\left(u+c_{1} z-x, t+z\right) \mathrm{d} H_{1}(x),
$$

the operator $\Phi$

$$
(\Phi \delta)(u, t)=\sup _{r \geq 0} \phi_{\delta}(r, u, t) \in B^{0}
$$

and conclude that there exists a function $r_{\delta}(u, t)$ such that $(\Phi \delta)(u, t)=\phi_{\delta}\left(r_{\delta}(u, t), u, t\right)$ for each $\delta \in B^{0}, u \in \mathbb{R}, t \in \mathbb{R}^{+}, r \in \mathbb{R}^{+}$.

The function $\phi$ and the operator $\Phi$ have the same properties as $\tilde{\phi}$ and $\tilde{\Phi}$ respectively, therefore we can formulate the solution of the fixed number claims problem in the following way.

THEOREM 4.2. The stopping time $\tau_{n, L}^{*}=T_{\eta_{n, L}}+R_{\eta_{n, L}}^{*}$ for $n=0,1, \ldots, L$, where

$$
\begin{aligned}
R_{i}^{*} & =r_{L-i}\left(U_{1}\left(T_{i}\right), T_{i}\right) \\
\eta_{n, L} & =L \wedge \inf \left\{i \geq n: R_{i}^{*}<S_{i+1}\right\},
\end{aligned}
$$

is optimal in the class $\mathcal{T}_{n, L}$. For this $\tilde{\tau}_{n, L}^{*}$, we have $\Gamma_{n, L}=E\left(J\left(\tau_{n, L}^{*}\right) \mid \mathcal{F}_{n}\right)$ and for $n=0$, we have $\Gamma_{0, L}=E\left(J\left(\tau_{L}^{*}\right) \mid \mathcal{F}_{0}\right)=\gamma_{L}(a, 0)$.

\subsection{Infinite number of claims}

In this section, we formulate the final solution of our problem. To this end, the additional assumption is needed. 
Assumption 3. $F_{1}\left(t_{0}\right)<1$.

The solution of the double stopping problem defined in equation (8) is following.

THEOREM 4.3. If the function $g$ and the distribution $F_{2}$ fulfill the assumptions of Theorem 3.4, $F_{1}$ fulfils Assumption 3 and has the density function $f_{1}$, then

(i) for $n \in \mathbb{N}$, the limit $\tau_{n}^{*}=\lim _{L \rightarrow \infty} \tau_{n, L}^{*}$ exists and $\tau_{n}^{*}$ is an optimal stopping rule in the set $\mathcal{T} \cap\left\{\tau \geq T_{n}\right\}$

(ii) $E\left(J\left(\tau_{n}^{*}\right) \mid \mathcal{F}_{n}\right)=\mu_{n} \gamma\left(U_{1}\left(T_{n}\right), T_{n}\right)$ a.s., where $\gamma$ is the uniform limit of $\gamma_{j}$ given by equation (20).

This follows by the same method as in Section 3. The function $\tilde{\gamma}(u, t)$ is bounded, continuous, nondecreasing with respect to $u$ and it has finite right-hand derivatives with respect to $u$ and $t$. We define the Markov process $\xi(t)=\left(t, U_{1}(t), Y(t), V(t)\right)$, where $Y(t)=t-T_{N_{1}(t)}, V(t)=\mu_{N_{1}(t)}$, with the state space $\mathbb{R}^{+} \times \mathbb{R} \times \mathbb{R}^{+} \times\{0,1\}$. We express the process, given by equation (16), as $J(t)=\tilde{g}(\xi(t))$ and calculate the strong generator of $\xi(t)$

$$
(A \tilde{g})(t, u, y, v)=v\left\{c_{1} \frac{\partial \tilde{\gamma}(u+, t)}{\partial u}+\frac{\partial \tilde{\gamma}(u, t+)}{\partial t}+\frac{f_{1}(y)}{\bar{F}_{1}(y)}\left[\int_{0}^{U} \tilde{\gamma}(u-x, t) \mathrm{d} H_{1}(x)-\tilde{\gamma}(u, t)\right]\right\} .
$$

The domain of $A$ contains all bounded functions, which have finite right-hand side derivatives in the first two coordinates. On the basis of Lemma 4.3, we conclude that $\tilde{g}$ belongs to the domain of the strong generator $A$. Let us notice, that there is only one difference in the form of strong generator of the process $\xi(t)$ in comparison with the generator of $\xi(s, t)$. It is the derivative with respect to $t$. It follows that the rest of the proof runs similarly as in Theorem 3.4.

Finally, using equation (8) the solution of double stopping problem is given by

$$
E Z\left(\tau^{*}, \tilde{\tau}^{*}\right)=E J\left(\tau^{*}\right)=\gamma\left(U_{1}(0), 0\right)=\gamma(a, 0)
$$

where $\tau^{*}$ and $\tilde{\tau}^{*}$ are defined according to Theorems 3.4 and 4.3.

\section{Final remarks}

The multiple stopping models are recently willingly applied as a tool of modelling the technical and economical phenomenon. The double disorder problem for the discrete time Markov processes has been investigated by Szajowski [21] and the multiple disorder of the continuous time processes by Nikolaev [15]. In mathematical finance, the multiple stopping approach is used by Carmona and Touzi [4] for modelling the swing option. Several models connected with multiple stopping have been considered in the area of yield management. Feng and Xiao [7] solved the problem of optimal pricing of inventories with multiple predetermined prices and Karpowicz and Szajowski [12] have considered the problem with possibility of double reversible price changes under the general predetermined price structure. Even though the process of sale in the problems of optimal pricing is similar to the risk process however there are important differences, such that the another techniques to solve them are used.

The presented model is a new approach to the risk reserve processes management. Its importance concerns both the applicability of the model and theoretical investigation on the 
optimization techniques in stochastic environment. Further research should be done in many directions to extend the risk model and possibilities of its applications. (cf. Muciek and Szajowski [14]).

\section{Acknowledgements}

The authors are grateful to an anonymous referee for pointing out errors in an earlier draft and for several suggestions that improved the presentation of this paper.

\section{References}

[1] Azuce, P. and Roberts, N., 2005, Optimal reinsurance and dividend distribution policies in the CramérLundberg model, Mathematical Finance, 15, 261-308.

[2] Boshuizen, F.A. and Gouweleeuw, J.M., 1993, General optimal stopping theorems for semi-Markov processes, Advances in Applied Probability, 4, 825-846.

[3] Brémaud, P., 1981, Point Processes and Queues, Martingale Dynamics (New York: Springer).

[4] Carmona, R. and Touzi, N., 2003, Optimal multiple stopping and valuation of swing options, Mathematical Finance, http://www.orfe.princeton.edu/rcarmona/download/fe/swing2.pdf. 32.

[5] Davis, M.H.A., 1993, Markov Models and Optimization (New York: Chapman and Hall).

[6] Eidukjavicjus, R., 1979, Optimalna ostanovka markovskoj cepi dvumia momentami ostanovki, Litovskii Matematicheskii Sbornik, 13, 181-183.

[7] Feng, Y. and Xiao, B., 2000, Optimal policies of yield management with multiple predetermined prices, Operations Research, 48(2), 332-343.

[8] Ferenstein, E.Z. and Sierociński, A., 1997, Optimal stopping of a risk process, Applicationes Mathematicae, 24(3), 335-342.

[9] Gikhman, I.I. and Skorokhod, A.V., 1973, The Theory of Stochastic Processes. II: Translated from the Russian by S. Kotz. Reprint of the First Edition 1973 (Berlin: Springer), vii, p. 441.

[10] Haggstrom, G.W., 1967, Optimal sequential procedures when more then one stop is required, The Annals of Mathematical Statistics, 38, 1618-1626.

[11] Jensen, U., 1997, An optimal stopping problem in risk theory, Scandinavian Actuarial Journal, 2, 149-159.

[12] Karpowicz, A. and Szajowski, K., 2005, Double optimal stopping times and optimal pricing model, Preprint, XN I-18/05/P-047, pp. 14.

[13] Muciek, B., 2002, Optimal stopping of a risk process: model with interest rates, Journal of Applied Probability, 39, 261-270.

[14] Muciek, B. and Szajowski, K., 2003, Optimal stopping of a risk process when claims are covered immediately, Preprint, http://neyman.im.pwr.wroc.pl/szajow/publ2002/pdf/OSRisk8i2006.pdf, submitted , pp. 12

[15] Nikolaev, A.F., 1998, On a formulation of the multiple "disorder" problem, Theory of Probability and Its Applications, 43(2), 293-297.

[16] Nikolaev, M.L., 1979, Obobshchennyje posledovatelnyje procedury, Litovskii Matematicheskii Sbornik, 191, $35-44$.

[17] Nikolaev, M.L., 1998, Optimal multi-stopping rules, Obozrenie Prikladnoj i Promyshlennoj Matematiki, 5(2), 309-348.

[18] Rolski, T., Schmidli, H., Schimdt, V. and Teugels, J., 1998, Stochastic Processes for Insurance and Finance (Chichester: John Wiley \& Sons).

[19] Schöttl, A., 1998, Optimal stopping of a risk reserve process with interest and cost rates, Journal of Applied Probability, 35, 115-123.

[20] Stadje, W., 1985, On multiple stopping rules, Optimization, 16, 401-418.

[21] Szajowski, K., 1996, A two-disorder detection problem, Applicationes Mathematicae, 24(2), 231-241. 
Copyright of Stochastics: An International Journal of Probability \& Stochastic Processes is the property of Taylor \& Francis Ltd and its content may not be copied or emailed to multiple sites or posted to a listserv without the copyright holder's express written permission. However, users may print, download, or email articles for individual use. 